\title{
Monitoring of biomass at Cooling Water System of hongyanhe nuclear power plant by using acoustic methods
}

\author{
WANG Zi ${ }^{1,2}$, TANG Yong ${ }^{1}$, FU Yuanyuan ${ }^{1}$, MENG Wei ${ }^{1,2}$, WANG Shuai ${ }^{2}$, LIU Xiaolin ${ }^{2}$ \\ ${ }^{1}$ Dalian Ocean University, College of Marine Sciences, Dalian116021,China \\ ${ }^{2} \mathrm{Su}$ Zhou Nuclear Power Research Institute, Suzhou 215004,China
}

\begin{abstract}
In recent years, there have been large-scale jellyfish outbreaks in the Bohai Sea in summer, which have greatly affected the water intake for cold source of Hongyanhe nuclear power station in Fuzhou bay and threatened the normal operation. This study aims at early warning of invasion of jellyfish, designs and develops acoustic monitoring system for cold source biological (jellyfish)by using ascientificechosounder (EY60,70kHz,Simrad), and carries out real-time monitoring of the echo of cold source biological entering water intake, so as to obtain the resource density of jellyfish from June to September 2019. The acoustic data is processed with a software of Echoview to calculate the average SV and detect the strength of the single target to calculate the daily average flux of the monitoring point. According to the monitoring results, the maximum and minimum daily average values of SV were $-62.7 \mathrm{~dB}$ and $-80.0 \mathrm{~dB}$, respectively. The maximum and minimum daily average values of target strength are $-37.2 \mathrm{~dB}$ and $-81.0 \mathrm{~dB}$, respectively. The maximum and minimum daily average values of flux are $5.36 \mathrm{ind} /(\mathrm{s} \cdot \mathrm{m} 2)$ and $1.2 \times 10-5 \mathrm{ind} /(\mathrm{s} \cdot \mathrm{m} 2)$.Combined with the daily cleaning amount of the monitoring point back-end intercepting network and meteorological data, the comparative analysis is carried out to determine the index level of the acoustic warning for cold source biological flowing into the water intake according to the correlation between average flux and amount of cleaned net.
\end{abstract}

\section{Introduction}

Recirculating cooling water system in coastal nuclear power plants and cooling water supply for other essential equipment are usually set at inshore water intake, and organisms entering the cooling water system from water intake are called cold-source organisms, defense, intercept and disposal of which will have a direct impact on safe and stable operation of the power plant. According to data statistics of American Institute of Nuclear Power Operations (INPO), the number of intake blocking events taking place in global nuclear power plants reached 61 from 2004 to 2008 , where nearly $80 \%$ of the events led to power reduction of generator set or shutdown, and over $20 \%$ of the events directly impacted safety systems of nuclear plants, and invasion of exotic cold-source organisms was the primary cause for intake blocking ${ }^{[1]}$. Cold source-induced organisms in domestic coastal nuclear power plants mainly include all kinds of jellyfishes, Acetes Chinensis and kelps, etc., especially the influence brought by outbreak of various types of jellyfishes in coastal areas is especially severe in recent years, seriously threatening safety production of coastal nuclear power plants in China. Hongyanhe Power Plant is located on the coast at the southeast side of Bohai Gulf, the ocean current in the intake area is mainly influenced by south and north reversing currents, and the outbreak of aurelia and Stomolopus nomurai in summer will generate intense cold-source organism impact on the water intake with the tidal flow. The existing arresting net-based cleaning and netting gear-based fishing can provide rough information about quantity of jellyfish resources, but when manual salvage and net cleaning operations fail under harsh sea conditions, it is impossible to give early warning of cold-source organisms, thus influencing the selection of effective responding and treatment measures and threatening safety operation of power plants.

Restricted by jellyfish observation and sampling technologies in the monitoring work of jellyfish resources, traditional monitoring work of large-scale jellyfishes mainly relies on netting gear sampling method and visual observation method, and jellyfish distribution and resource variation are investigated through netting gear-based fishing or visual observation results. This method is characterized by unified sampling method and convenient operation, and moreover, it can intuitively reflect planar distribution characteristics of large-scale jellyfishes. When it comes to its defect, it lacks intuitive judgment and analysis data regarding underwater jellyfish swimming, colony distribution and vertical movement laws. With rapid development of ocean exploration technologies in recent years, underwater acousto-optical technology and air photo techniques have already been applied to jellyfish monitoring and survey. Davis et al. researched and

\footnotetext{
${ }^{*}$ MENG Wei: mengwei@cgnpc.com.cn
} 
developed an underwater plankton video recording system via underwater optical technique and realized imaging of zooplanktons using forward scattered light, which could measure zooplanktons within $0.2-20 \mathrm{~mm}$, such as polyps and jellyfishes, etc.; Graham et al. developed a jellyfish image pickup system to realize underwater in-situ optical monitoring of jellyfishes ${ }^{[12]}$; Liansheng $\mathrm{Yu}$ et al. realized automatic field shooting of zooplankton images using a "full-automatic digital micro-imager" ${ }^{\prime[13]}$. Although underwater optical image pickup results are intuitive, they are still restricted by objective factors like turbid water body and detection field. Houghto et al. employed air photo technique to monitor three types of large-scale jellyfishes in South Ireland area, these jellyfishes had large bodies (diameter was greater than $1 \mathrm{~m}$ ) with different opaque colors, and they could be directly identified through naked eyes at low altitude $(152 \mathrm{~m})^{[14]}$. In comparison with underwater optical technology, horizontal monitoring range of aerial imaging technology is large, but it can only monitor water bodies at surface layer or near surface layer, and it is applicable to monitoring of distribution status and movement \& migration behaviors of large-scale jellyfishes, etc.

With rapid development of underwater sound techniques in fishery industry in recent years, carrying out fixed-point acoustic monitoring of migratory fish resources in water areas with great liquidity has become an important work content in ecological protection of rivers in international countries with developed fishery industry. The main features are broad detection zone, possible detection in turbid or dark water bodies, continuous spatial and temporal distribution information, etc. Kang et al. used a split-beam scientific fisher finder (38 kHz and $120 \mathrm{kHz}$ ) to determine target strength of large-scale Stomolopus nomurai in coastal net cages in Korean water area, established the model relationship between umbrella diameter and target strength of Stomolopus nomurai and found that target strength of Stomolopus nomurai presented linear growth with increase of umbrella diameter under two detection frequencies, and the results could be applied to acoustic evaluation of Stomolopus nomurai resources ${ }^{[15]}$. Yoon et al. used frequency difference $(38 \mathrm{kHz}$ and $120 \mathrm{kHz})$ method to conduct acoustic identification \& classification and resource evaluation of jellyfishes in Korean water area in 2019[16].

Therefore, summer jellyfishes at water intake of Hongyanhe Nuclear Power Plant were taken as monitoring objects, an acoustic monitoring and early warning system based on split-beam scientific fish finder was designed and developed to perform real-time acoustic monitoring of cold-source organisms like jellyfishes which entered the sea area of arresting net, and calculate and analyze jellyfish density and flux, so as to lay a scientific foundation for early warning of jellyfish biomass entering water intake.

\section{Materials and methods}

\subsection{Monitoring position and sonar}

As shown in Fig. 1, five arresting nets were totally set water intake of Hongyanhe Nuclear Power Plant, and the outmost arresting net was fixed using float bowls and anchor blocks, so the conditions for sonar setting were not satisfied. Hence, sonar monitoring point in this study was set as the first caisson (4\# caisson) at the south side before the second outside arresting net, shore-based monitoring method was used, the transducer of the splitbeam scientific fish finder $(\mathrm{EK} 60,70 \mathrm{kHz}, \mathrm{Simrad}$, Norway) was fixed and installed in underwater horizontal direction, and the installation position and beam direction are shown in Fig. 1.The transceiver (GPT) of the scientific fish finder was installed in the power distribution cabinet set on the caisson platform, control signal and data transmission of GPT were passed back to the industrial personal computer in the shore-end control room through the erected cables, thus realizing remote operation and control of the scientific fish finder, and the main parameters in fixed-point monitoring were: transmitting power $700 \mathrm{~W}$,transceiving cycle $1 \mathrm{~s}$ and pulse width $0.512 \mathrm{~ms}$.

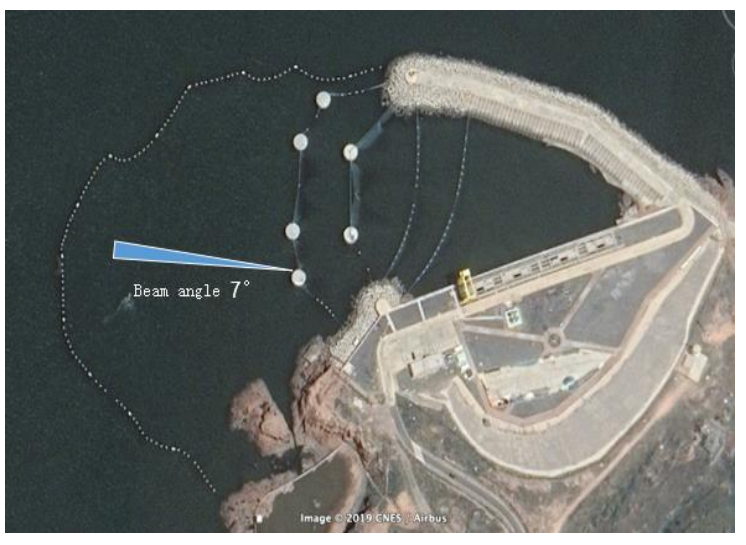

Fig.1 The location of sonar monitoring system in the water intake of Hongyanhe Nuclear Power Plant

\subsection{Composition of sonar monitoring system}

Fig. 2 shows the composition of the sonar monitoring system, which is mainly divided into three parts: frontend equipment unit, shore-based control processing unit and client application unit, where front-end equipment unit is located on caisson, and it is mainly responsible for data acquisition, including scientific fish finder system, overwater camera and multifunctional meteorograph, etc.; The shore-based control processing unit mainly includes industrial personal computer and server, and it acquires and saves data on the industrial personal computer and transmits the acquired data to the server for saving and processing via network; The application unit mainly displays sonar echo graph and computational analysis results, including display of monitoring information and early warning at fixed client end and mobile client end, etc. 


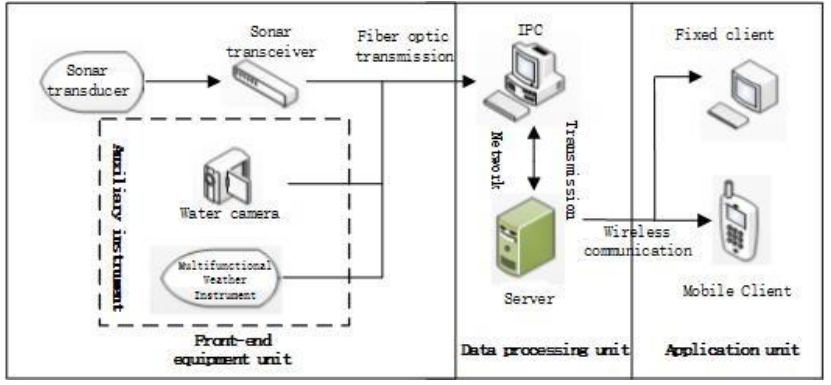

Fig.2 Schematic diagram of sonar monitoring system in the water intake of Hongyanhe NuclearPower Plant

\subsection{Sonar transducer setting}

The transducer of scientific fish finder was installed on the designed and developed special stand, fixed at outer side of 4\# caisson (Fig. 1), connected in the power distribution cabinet on the caisson and connected to the transceiver. Fig. 3 shows the design structure diagram of transducer stand. This stand could manually adjust horizontal azimuth angle and vertical dip angle of the transducer, the facing direction (namely beam direction) of the transducer was set as perpendicular to the crosssectional direction of water intake, and the depth was set as $0.5 \mathrm{~m}$ below the maximum low water. The sonar monitoring area was coverage area (restricted by tide level range) of wave beam (beam angle $7^{\circ}$ ) at $100 \mathrm{~m}$ or so before the water intake. The transducer's orientation and dip angle were adjusted on the field according to sonar echo graphs, it was determined at northwest $278^{\circ}$ in horizontal direction, and this direction was in the direction of the intake jellyfishes entered; Its vertical dip angle was upward $1.2^{\circ}$. Through the echo graph, it was confirmed that effective monitoring distance was $60 \mathrm{~m}$.

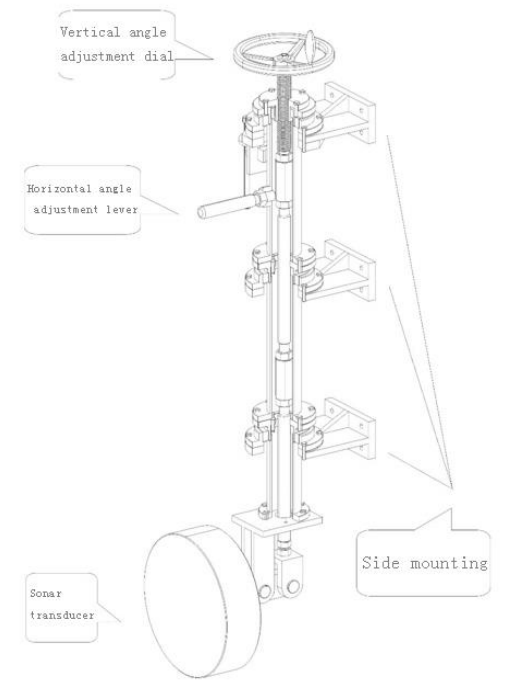

Fig.3 Design diagram of transducer bracket with adjustable horizontal and vertical angle

\subsection{Overwater camera and meteorological monitoring}

As fishing net boats passed the front end of sonar beam in the area of arresting nets, wake bubbles of these fishing net boats would form strong disturbing echo, and then an overwater camera was set to perform real-time monitoring of these boats, and meanwhile, the sea conditions could also be monitored to provide information for echo identification. A multifunctional meteorological monitoring station was set on the caisson to provide meteorological information like wind speed, wind direction, air temperature and humidity in a realtime way.

\subsection{Shore-based control and user service}

The industrial personal computer set in the shore-based monitoring room was connected to sonar GPT via photoelectric composite cable, which could realize realtime control and display of working conditions of sonar and camera as well as data storage and online uploading, where sonar monitoring was implemented using the display \& control software ER60 carried by the scientific fish finder, and the overwater camera conducted realtime monitoring via the supporting software. Monitoring data were transmitted to the server of Suzhou Thermal Power Research Institute through outer net for data storage and model calculation, and timely acquired information and related early warning information were provided to fixed and mobile client ends through the development software.

\subsection{Sonar monitoring and data processing methods}

The installation work was completed and monitoring work was started on May 25, 2019. Real-time monitoring of cold-source organisms like jellyfishes flowing into intake was carried out. The acoustic data were processed with a special software Echoview4, and after bubble echo under high tide condition and wake bubble echo of passing boats were excluded, integral processing of volumetric scattering intensity and monomer target detection were implemented. The SV integral threshold was set as $-70 \mathrm{~B}$, and the detection range of echo integral and monomer target was within effective acoustic detection range $(1 \mathrm{~m}-60 \mathrm{~m})$. According to jellyfish specifications of cleaning net, the minimum detection threshold was set as $-80 \mathrm{~dB}$, followed by daily weighted averaging of TS of monomer targets. On this basis, daily average values of SV and TS were acquired, and daily average density of jellyfishes passing through the sonar beam was calculated,

$$
<n>=<s_{V}>/<\sigma_{b s}>
$$

Where $\mathrm{sV}$ is reverse volumetric scattering coefficient (unit: $\mathrm{m}^{2} / \mathrm{m}^{3}$ ), and the relationship with SV (unit: $\mathrm{dB}$ ) is:

$$
S_{V}=10 \log s_{V}
$$

obs is reverse scattering cross section (unit: $\mathrm{m}^{2}$ ) and its relationship with TS (unit: $\mathrm{dB}$ ) is:

$$
\sigma_{b s}=10^{T S / 10}
$$

Given this, daily average flux of organisms passing through sonar beam is calculated 


$$
<\boldsymbol{F}>=\langle n>|v| \times \sin \theta
$$

Where $\mathrm{v}$ is flow velocity at intake and $\theta$ is the included angle between flow velocity and cross section of intake. The flux unit is ind/(s $\left.\cdot \mathrm{m}^{2}\right)$.

\subsection{Net cleaning capacity of jellyfishes}

Net cleaning capacity of jellyfishes was acquired from daily report of the nuclear power plant, and it was the weight of jellyfishes and other sundries cleaned by trash holding and cleaning workers in different arresting nets every day. As the cleaning work of arresting nets at water intake was interrupted because of sea conditions and due to the influence of visual observation and estimation of operators, week was taken as unit for statistical analysis.

\section{Results and analysis}

\subsection{Sonar monitoring results}

Fig. 4 shows the acoustic echo image maps of actually monitored cold-source organisms flowing into sonar beam. The effective monitoring range was $60 \mathrm{~m}$ or. Influenced by tidal level and water depth, effective monitoring range of organisms under high tidal level could reach $90 \mathrm{~m}$ (Fig. 4-A). The red linear echo at 120 $\mathrm{m}$ is echo from outside arresting net; The red linear echo at $160 \mathrm{~m}$ is echo from distant sea bottom; Blue and green echoes are sidelobe echoes on sea surface and sea bottom.

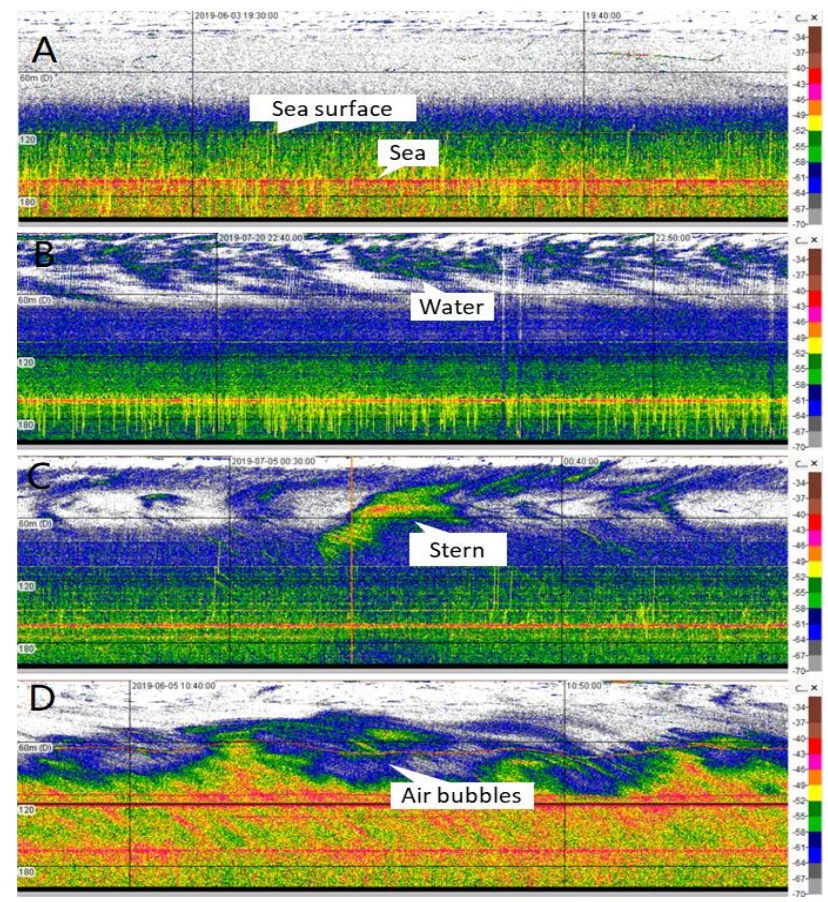

Fig.4 Echogram of sonar monitoring systemfixed-point: A: low biological density echo; B: high biological density echo; C: stronger bubble echo of passing ships; D: echo of sea bubbles in high sea states.

\subsection{Echo identification of boat wake flow}

The left and right pictures in Fig. 5 are sonar echo graphs of two boats (numbered as 1 and 2) before sonar beam and images shot by the overwater optical camera, respectively. Before passing through the sonar transducer, $1 \#$ boat was about $10 \mathrm{~m}$ from the transducer while $2 \#$ boat was about $120 \mathrm{~m}$ from the transducer.

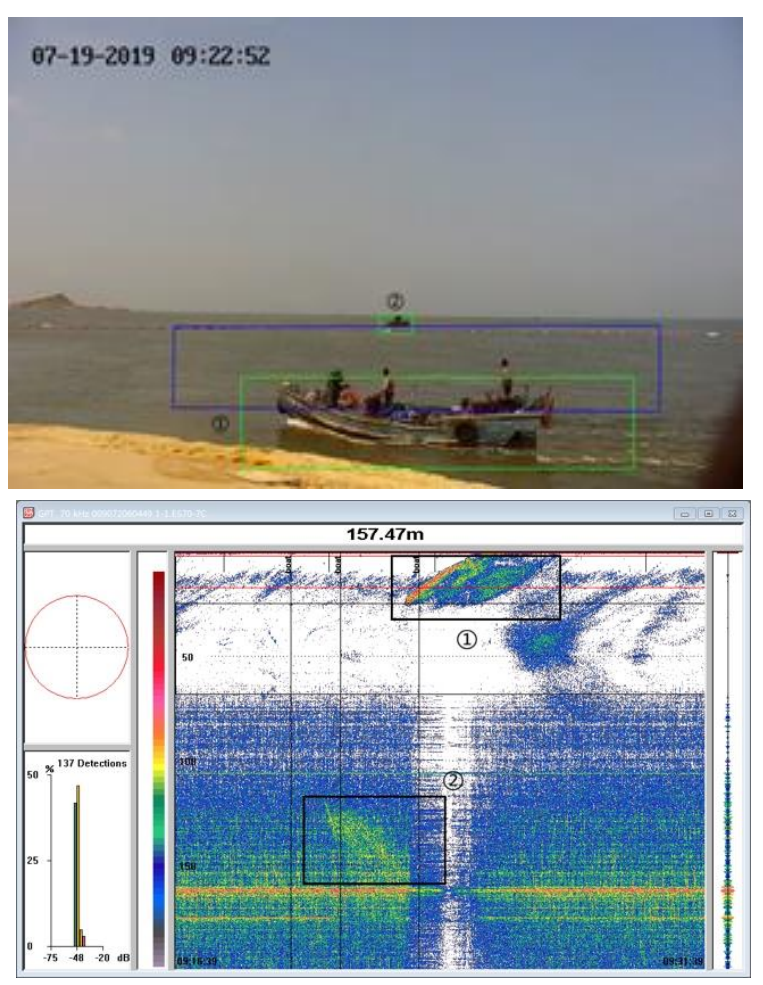

Fig.5 Vessel wake echoes confirmed by water camera

\subsection{Flow velocity at intake}

Flow velocity data at water intake was previously measured value of Hongyanhe Nuclear Power Plant, and average flow velocity under spring tide was $0.5 \mathrm{~m} / \mathrm{s}$, which under moderate tide was $0.26 \mathrm{~m} / \mathrm{s}$ and that under neap tide was $0.01 \mathrm{~m} / \mathrm{s}$.

\subsection{Average SV and average TS}

The monitoring and statistical results of daily average SV and TS from June to July in 2019 are displayed in Fig. 6. The maximum SV value appeared on June 8, being $-62.5 \mathrm{~dB}$; The minimum value appeared on July 20 , being $-76.3 \mathrm{~dB}$; The maximum value of average target strength appeared on June 6, being $-37.2 \pm 19.1 \mathrm{~dB}$, and the number of detectable and traceable monomer targets was 3,562; The minimum value appeared on June 22, being $-81.1 \pm 3.1 \mathrm{~dB}$, and number of detectable and traceable monomer targets was 44 . 


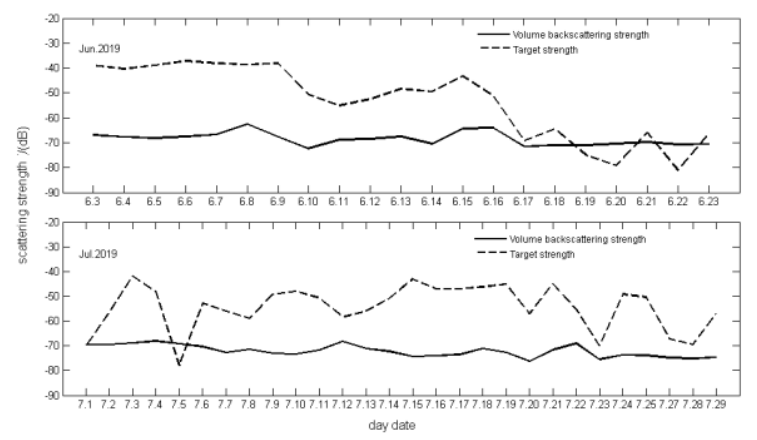

Fig.6 Daily average SV and TS of Jellyfish througt the water intake of Hongyanhe Nuclear Power Plant in Jun., and Jul.2017

\subsection{Jellyfish flux}

Daily average flux from June to July was calculated according to formula (4), and the result was shown in Fig. 7. Large peak values appeared on June 20 and 22 and July 5 , being $1.97 \mathrm{ind} /\left(\mathrm{s} \cdot \mathrm{m}^{2}\right), 5.36 \mathrm{ind} /\left(\mathrm{s} \cdot \mathrm{m}^{2}\right)$ and $3.62 \mathrm{ind} /\left(\mathrm{s} \cdot \mathrm{m}^{2}\right)$, respectively. The maximum value appeared on June 22, and average flux was $5.36 \mathrm{ind} /\left(\mathrm{s} \cdot \mathrm{m}^{2}\right)$; The minimum value appeared on June 5 , and average flux was $1.2 \times 10-5 \mathrm{ind} /\left(\mathrm{s} \cdot \mathrm{m}^{2}\right)$.

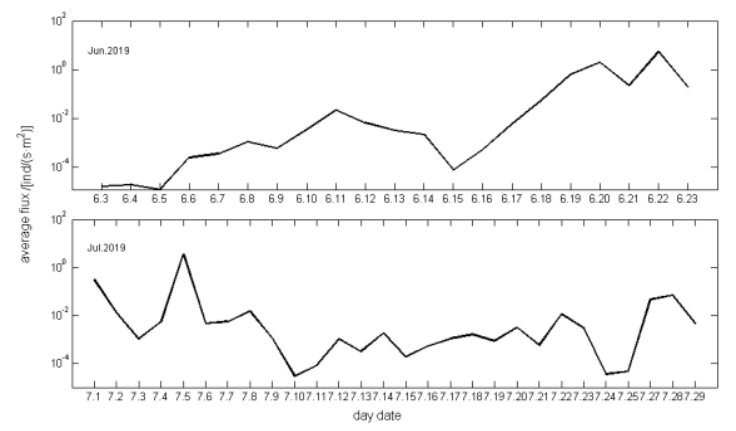

Fig.7 Flux of Jellyfishin the water intake of Hongyanhe NuclearPower Plant in Jun., and Jul.2017

\subsection{Flux and net cleaning capacity}

The comparison results of average flux and net cleaning capacity by weeks are shown in Fig. 8 . The 1 st to $8^{\text {th }}$ weeks represented eight weeks from June to July. The peak average flux appeared from the 3rd to 5th week, and the maximum value was $8.43 \mathrm{ind} /\left(\mathrm{s} \cdot \mathrm{m}^{2}\right)$; The peak net cleaning capacity appeared in the $4^{\text {th }}$ and $5^{\text {th }}$ weeks, where the maximum value was 56t. The minimum values of both average flux and net cleaning capacity appeared in the first week, being $0.002 \mathrm{ind} /\left(\mathrm{s} \cdot \mathrm{m}^{2}\right)$ and $0.4 \mathrm{t}$, respectively.

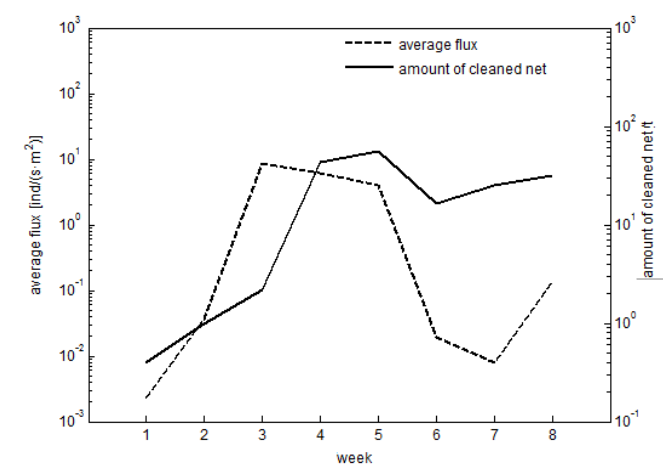

Fig.8Jellyfish flux and amount of cleaned net in the water intake of Hongyanhe Nuclear Power Plant in Jun,Jul.2017

\section{Discussion}

For the outbreak of cold-source organisms in coastal nuclear power plants, a sonar shore-based monitoring system based on a split-beam scientific fish finder (EY60) was developed in this paper. According to two-month experimental monitoring results at water intake of Hongyanhe Nuclear Power Plant, the system had good monitoring \& early earning function and actual application prospect, specifically discussed as follows:

\subsection{Use of horizontal sonar beam}

The split-beam scientific fish finder is a professional instrument realizing acoustic survey and evaluation of fishery resources with narrow $7^{\circ}$ half-power beam angle, and as a general rule, the beams are used to detect fishery resources in a vertically downward way. As the designed water depth at the intake of coastal nuclear power plant is generally shallow (10 $\mathrm{m}$ or so), to enlarge acoustic sampling volume of cold-source organisms, beam detection mode in horizontal direction was used, the effective detection range exceeded $60 \mathrm{~m}$, and the echo graphs of cold-source organisms were intuitive and obvious (Fig. 4), thus laying a foundation for realizing electric stepping rotation in horizontal direction and further expanding the acoustic monitoring range. However, as horizontal use of beams would generate different target strength of cold-source organisms in horizontal direction, spatial statistics of average target strength of attitudes should be considered in a 3D coordinate system. A more detailed study regarding this aspect should be carried out in the future by combining types of cold-source organisms and flow field change at intake, etc.

\subsection{Evaluation of quantity of cold-source biological resources}

The acoustic monitoring system of cold-source organisms in the nuclear power plant in this study was developed based on monomer target detection and echo integrating functions of the split-beam scientific fish finder, and it could monitor the density of cold-source biological resources (Fig. 6). On this basis, the flow velocity at intake was combined to measure the flux of 
cold-source organisms, and early warning grade indexes were provided. The cold-source organisms in the intake area of Hongyanhe Nuclear Power Plant were mainly jellyfishes in summer. In the current monitoring experiment, through the comparison with net cleaning capacity data of organisms like jellyfishes, average flux of jellyfishes had a good correlation tendency with jellyfish weight in cleaning net, and the jellyfish biomass monitoring effect was very good (Fig. 8), but the body length relation and weight relation corresponding to average target strengths of attitudes of jellyfishes (aurelia and Stomolopus nomurai) should be established in order to evaluate the quantity (wet weight) of coldsource biological resources passing through the intake. Meanwhile, acoustic identification of target echoes of other cold-source species should be further investigated.

\subsection{Flow velocity at intake}

Average flux parameter of jellyfish resources was mainly used in this study for grade assessment of early warning indexes related to impact of cold-source organisms on intake of nuclear power plant (Fig. 7), so flow velocity at intake was also one of important early warning indexes. As flow velocity at intake of Hongyanhe Nuclear Power Plant was mainly influenced by tide, wind speed and wind direction, the dynamic flow velocity information at intake should also be mastered. The flow velocity parameters used in this study were average flow velocities of high, moderate and low tides, which were calculated according measured values in previous years, so they had certain deviations from daily actual situation, and further perfection was needed in the follow-up monitoring work.

\subsection{Sonar monitoring system}

The acoustic monitoring system designed and developed in this study basically satisfied the requirements for monitoring cold-source organisms in Hongyanhe Nuclear Power Plant, but it still had some problems needing improvements, mainly including:

1) Remote control problem of sonar transducer attitude

As sea conditions at intake, wind and rain and surface bubbles generated by passing boats will generate a great impact on the monitoring area (C and D in Fig. 4, stepper motor can be added onto the transducer stand to realize remote control of attitude angle of the transducer.

2) Transducer maintenance

In the long-time monitoring process in summer, fouling organisms will be adhered to the transducer, thus influencing the accuracy of acoustic detection, so it should be regularly cleaned and maintained by divers.

3) Dynamic data analysis

The currently developed monitoring system could only provide real-time and statistical SV data of the detection objects, jellyfish density data were processed with a professional software, and the automatic processing and analysis level was not reached yet. Therefore, it is necessary to develop monomer target species identification, specification presumption, density and wet weight evaluation and early warning grade index models for specific cold-source biological species, so as to provide an effective guarantee for the cause of coastal nuclear power in China.

\section{Acknowledgement}

This work is supported by National Key R\&D Program of China (2017YFC1404400-Subject 4 and 6)

\section{References}

1. Ruan Guoping. Analysis and countermeasures of water intake blockage in nuclear power plant [J]. Nuclear power engineering,2015,36(S1):151-154.

2. Brodeur R D,Mills C E,Overland J E,Walters G E, Schumacher J D,Evidence for a substantial increase in gelatinous zooplankton in the Bering Sea, with possible links to climate change,[J]Fisheries Oceanography,1999,8(4) : 296-306.

3. Purcell J E.Predation on zooplankton by large jellyfish,Aurelia labiate,Cyaneacapillata and Aequoreaaequorea,in Prince William Sound,Alaska,[J]Marine Ecology Progress Series,2003,246: 137-152.

4. Li Jiansheng,Ling Jianzhong,Cheng Jiahua,Li Huiyu. Analysis of the Resources of the Shahi-Amaranth in the North East China Sea in Summer and Autumn of 2008.[J] Marine fishery,2009.31( 4) : 444-449.

5. Wang Bin,Li Yulong,Shen Hong,Li Yiping,Wang Wenbo,Sun Ming,Dong Jing. Distribution of White Xia Jellyfish Offshore in North Liaodong Bay from 2005 to 2013.[J] Marine fishery,2014,36(2):146-154.

6. Randriarilala F,Kitakado T,Shiode D,Sakaguchi M,Hayashi T,Tokai T, Densityestimation of the giant jellyfish Nemopilemanomurai around Japan using an alternative modified detection function for left truncation in a line transect survey. [J] Fisheries Science,2014,80(2) : 261-271.

7. Wang Shiwei,Zhang Guangtao,Sun Song,Wang Yantao,Zhao Zengxia.Population dynamics of three large jellyfishes in Jiaozhou Bay in summer of 2011.[J] Oceans and lakes,2012,43(3):471-479.

8. Wang Bin,Fang Lichen,Dong Jing,Li Yulong,Liu Xiuze,Li Yiping,Sun Ming,Wang Wenbo. Research progress on acoustic observation and evaluation technology of large jellyfish [J] Acta Ecologica Sinica,2017,37(24):8187-8196. 